BMJ Open Sport \& Exercise Medicine

\title{
Comparison of tetradecyl sulfate versus polidocanol injections for stabilisation of joints that regularly dislocate in an Ehlers-Danlos population
}

\author{
Fraser Burling
}

To cite: Burling F. Comparison of tetradecyl sulfate versus polidocanol injections for stabilisation of joints that regularly dislocate in an EhlersDanlos population. BMJ Open Sport \& Exercise Medicine 2019;5:e000481. doi:10.1136/ bmjsem-2018-000481

Accepted 25 December 2018

\section{ABSTRACT}

Objectives To determine whether there is similarity between tetradecyl sulfate and polidocanol in stabilising a joint from dislocating in patients with Ehlers-Danlos syndrome (EDS).

Method A retrospective analysis of patients with EDS in a sole-practice clinic in New Zealand. Patients must have had the diagnosis of EDS, had easily dislocatable joints, had treatment and at least 3 months' follow-up. 0.11\% tetradecyl sulfate solution, or $0.25 \%$ polidocanol solution, was injected to ligament attachments (enthesis) on the side of the joints where they dislocated. Patients were deemed successfully treated if their affected joints were no longer dislocated over a minimum of 3 months' follow-up (out to 3 years).

Results 0 250 patients at the time of the study, 46 fitted the criteria. There were 37 treated with tetradecyl sulfate and nine with polidocanol. For the tetradecyl group there were a total of 305 injections around 97 joints: mean 3.1, range $1-22$, median 2 . For the polidocanol group there were 36 injections around 19 joints: mean 1.9, range 1-8, median 2. The difference of means between group 1 (tetradecyl) and group 2 (polidocanol) is 1.2 , Cls 0.34 to 2.98. All patients had no further dislocations of treated joints unless they had a major new injury (two patients). Conclusion There was no difference between the two groups for stabilising joints from dislocating. These two agents appear promising for treating patients with recurrent joint dislocations in the setting of EDS. Prospective multicentre randomised controlled trials are needed to confirm these data.

\section{INTRODUCTION}

Ehlers-Danlos syndromes (EDS) are a genetically heterogeneous population that are almost always hypermobile with variable abnormalities in multiple organs. EDS is primarily due to issues of abnormalities within collagen synthesis and binding. There are 30 known genes so far with both autosomal dominant and recessive genes being represented. About one-third of these patients have issues with joint dislocations (large and small joints) that can significantly affect their quality of life. The New York Criteria

\section{What are the new findings?}

While there are studies on the use of tetradecy sulfate and polidocanol in soft tissues, there are no studies to date on the use of these agents in treating chronic and recurrent dislocations in the EhlersDanlos population, or any population, other than for temporomandibular joints.

How might it impact on clinical practice in the near future?

Before being added to routine care tetradecyl sulfate will need to be studied in other trials with randomised controlled trial designs. A successful treatment could impact on the lives of tens of thousands of people with EDS internationally who are chronic sufferers from chronic and recurrent dislocating joints.

for EDS has summarised these issues well in the American Journal of Medical Genetics Part $C$, March 2017, with 18 articles. ${ }^{1}$ The use of steroid injections is relatively contraindicated but prolotherapy has been gaining ground in patients with EDS. ${ }^{23}$

Prolotherapy/sclerosant therapy has been well established as agents that lay down fibrous scar tissue: in varicose veins, ${ }^{4-7}$ haemorrhoids and soft tissue ${ }^{8}$ over more than seven decades. Prolotherapy use in soft tissue has been around for 60 years and was extensively pioneered by George Hackett, an American orthopaedic surgeon. ${ }^{9}$ The use of dextrose is widespread but we have found that it is not as efficacious as the stronger agents that are used in varicose veins-such as polidocanol and tetradecyl sulfate. Tetradecyl sulfate was first published as a vein sclerosant in 1946. ${ }^{10}$ There are over 1200 articles on the use of tetradecyl in both veins and soft tissue (Medline search 1946-2018). Polidocanol is also well established as a good sclerosant, also 


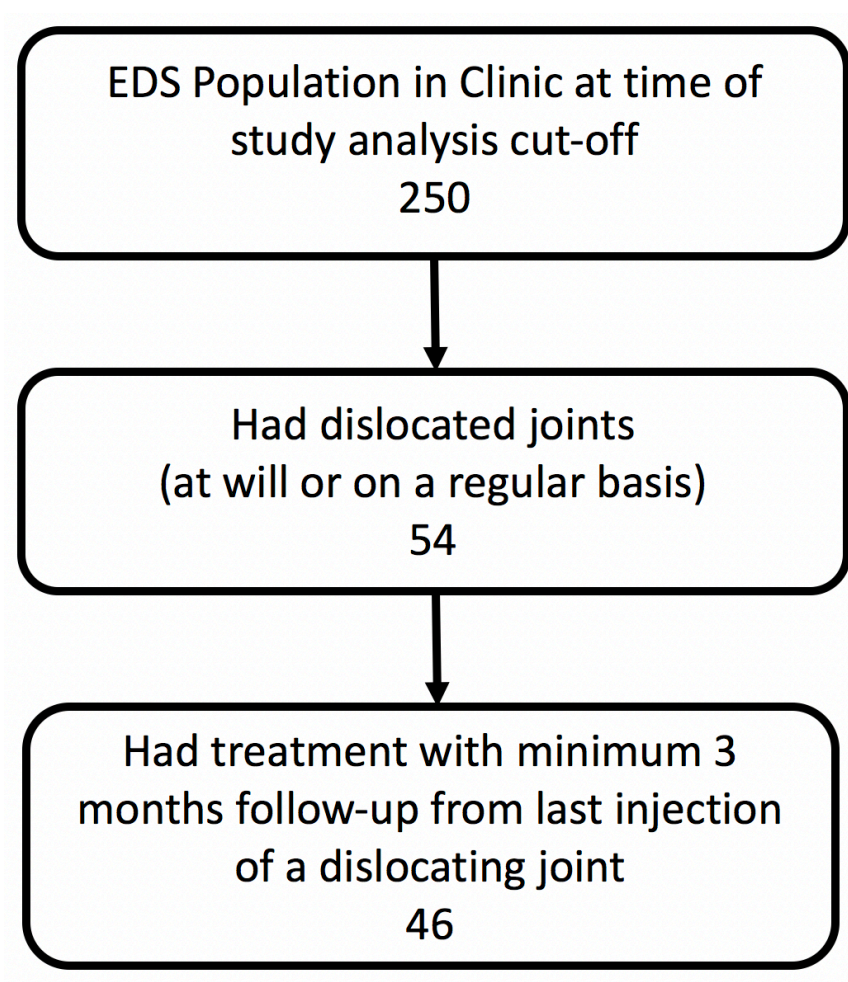

Figure 1 Flow diagram for patient selection. EDS, EhlersDanlos syndrome.

with over 1200 articles on its use in veins and soft tissue, from 1980 onwards. ${ }^{11}$

Many studies have appeared in the last 15-20 years on the efficacy of these stronger agents in soft tissue (including knee ligaments, ${ }^{12-14}$ elbow ligaments, ${ }^{15-18}$ shoulder rotator cuff insertions, ${ }^{19}$ fauces (for snoring and obstructive sleep apnoea $)^{20} 21$ and dislocating temporomandibular joints (TMJ)). ${ }^{22}$ Our clinic gave up on dextrose many years ago, even as a back-up for those with allergies to stronger agents, due to its lack of efficacy.

People with EDS have tissue that appears to be more easily damaged. We have noted that it requires less force with an accident or sporting injury to cause injury in the EDS group. If one can build up the tissue, instead of softening it with steroids then one may be able to stop dislocations.

It is easy to break individual sticks, but hard to break a whole bundle.

Likewise: It is easy to break a few strands of collagen, but hard to break a whole bundle (even abnormal collagen). If we can build up the bundle of collagen, then breaking the bundle becomes that much harder.

Prolotherapy has been used at our clinic since 1960 . While treating sources of pain in our patients with EDS we noted that we stopped them from dislocating. In recent years, we have been using the stronger agents, tetradecyl sulfate and polidocanol, and found them to be more successful than dextrose solutions. This study is the comparator between those agents.

\section{METHOD}

A retrospective analysis of all patients with EDS in a singlecentre, sole-practice clinic in New Zealand. To qualify for the study, the patients must have had EDS according to the New York Criteria, had easily dislocatable joints (either at will or on regular basis), had treatment and had at least 3 months' follow-up from last injection to determine that the joints are no longer dislocating; and for analysis all patients must also be aged 18 or over (figure 1).

Two millilitres of $0.11 \%$ tetradecyl sulfate solution, or $0.25 \%$ polidocanol solution, was injected to ligament attachments (enthesis) on the side of the joints where they dislocated. Dilution was with the anaesthetic prilocaine as it appears to have a much lower risk for side effects than xylocaine (lidocaine/lignocaine).

Patients' joints were deemed successfully treated if their affected joints were no longer dislocated over a minimum of 3 months' follow-up (out to 3 years). When a patient had more than one joint affected the follow-up is a minimum of 3 months from the last joint treated.

Important notes when treating: Treatments were only performed after fractures (if any) had healed. Injections were not given if a patient had an active infection. Dislocated joints were reduced before any injections were given. Polidocanol was used for any patient with a known sulfa allergy. Injections were given to any one area with a minimum of 2 weeks (mean 4 weeks) between injections if same spot was needing more. A maximum of four injections were given on any 1 day (due to limiting the anaesthetic dose to less than $3 \mathrm{mg} / \mathrm{kg}$ body weight).

The dislocated joints were treated with injections at the enthesis of the joint capsules and surrounding ligamentous and tendon structures. Examples are shown for the shoulder with treatment to the attachments of the subscapularis, supraspinatus (for anterior dislocations), infraspinatus and teres minor (for posterior dislocations) (figure 2). If the joint dislocated inferiorly on raising the shoulder then the teres major was treated with both an anterior and posterior approach (to avoid the nerves, arteries and veins running through the axilla). The hip mainly needed injection to the iliofemoral attachments and was reached with a 3.5-inch spinal needle (figure 3 ). The rib attachments are best approached from $3 \mathrm{~cm}$ out on the rib, where it is close to the surface, and then move along the surface of the rib, to avoid missing the rib and risking a pneumothorax (figure 4). The knee was most commonly treated at the inferior attachments of the medial collateral ligaments. The ankle was most commonly treated at the fibular-calcaneal, and fibular-talar attachments. The elbows were most commonly treated at the annular (radial sling) attachment; the lateral epicondyle rarely needed treatment. The TMJ was treated by injecting around the joint, not in the joint, and at the adjacent masseter attachment. Injections were performed using anatomical landmarks, and always in bone contact (some may prefer ultrasound imaging to assist). 

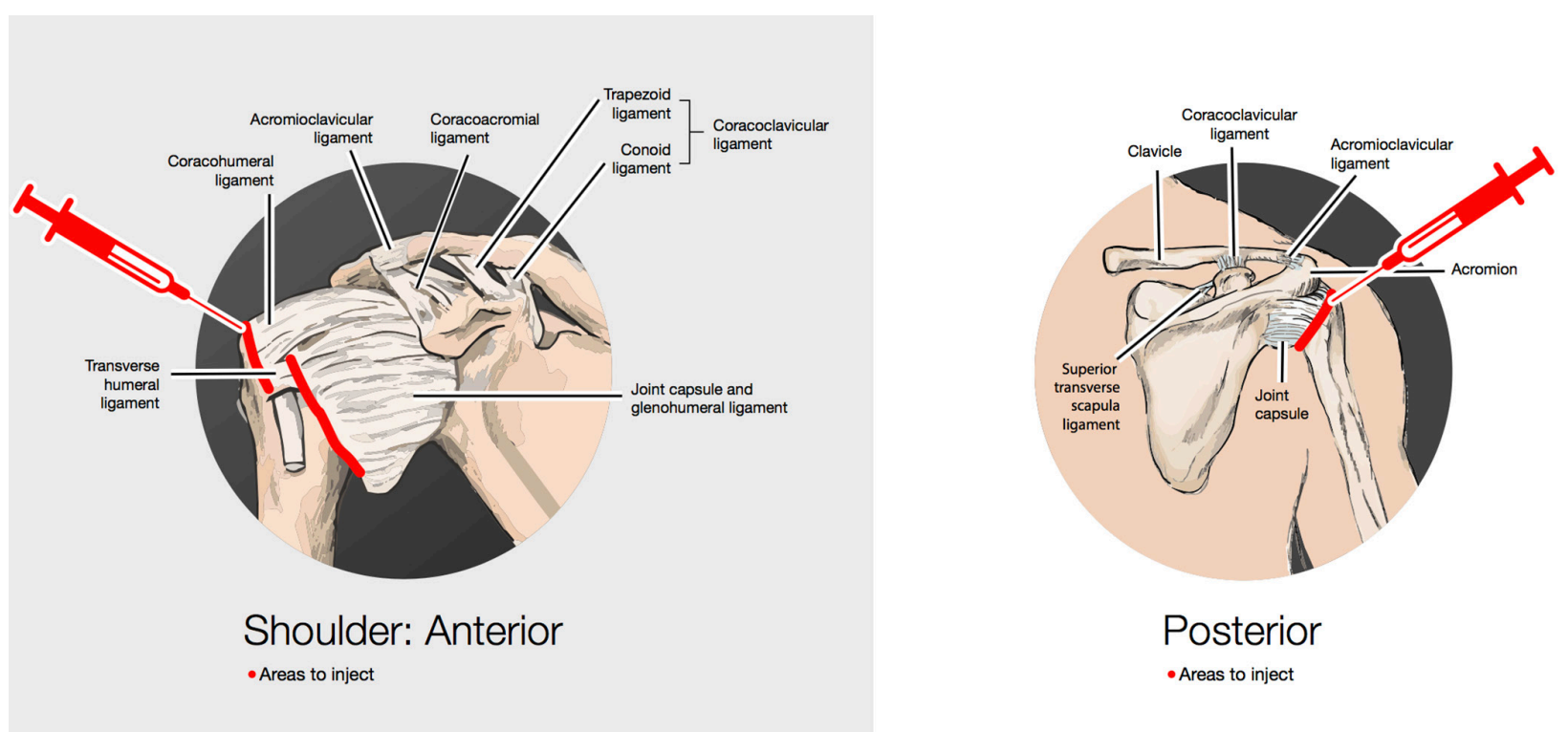

Figure 2 Examples for areas treated on a shoulder: injection is at the enthesis (in bone contact), on the side where the shoulder dislocates (some shoulders dislocated in both directions, but posterior dislocation was more common); and inject where it is tender (in red).

\section{RESULTS}

Of 250 patients at the time of the study, there were 46 who fitted the criteria: 15 were hypermobile EDS and 31 were classical EDS according to the New York Criteria (there was no difference in treatment options and outcomes for the subanalysis-data not published). The age range was 18-75 with one male and 45 females.

There were 37 who had been treated with tetradecyl sulfate and nine who were treated with polidocanol. (Only patients with a known sulfa allergy were treated with polidocanol, hence the reason for disparity in numbers treated in each group.) All patients had no further dislocations of treated joints unless they had a major new injury (two patients). (I always wait for a minimum of 6 weeks before treating new injuries, to allow for nature's healing first.)

For the tetradecyl group there were a total of 305 injections around 97 joints: mean 3.1, range 1-22, median 2 (shoulders, hips, knees, ribs, TMJs, elbows, ankles). For the polidocanol group there were 36 injections around 19 joints: mean 1.9, range 1-8, median 2 (shoulders, hips, knees, TMJs, ankles).

Student's unpaired t-test score gave a $\mathrm{p}$ value of 0.12 (statistical significance at $\mathrm{p}<0.05)$. The difference of means between group 1 (tetradecyl) and group 2 (polidocanol) is 1.2, CIs for this difference of the second mean from the first mean is -0.34 to 2.98 . Wilcoxon rank-sum two-tailed test for a non-standard distribution gave a $\mathrm{p}$
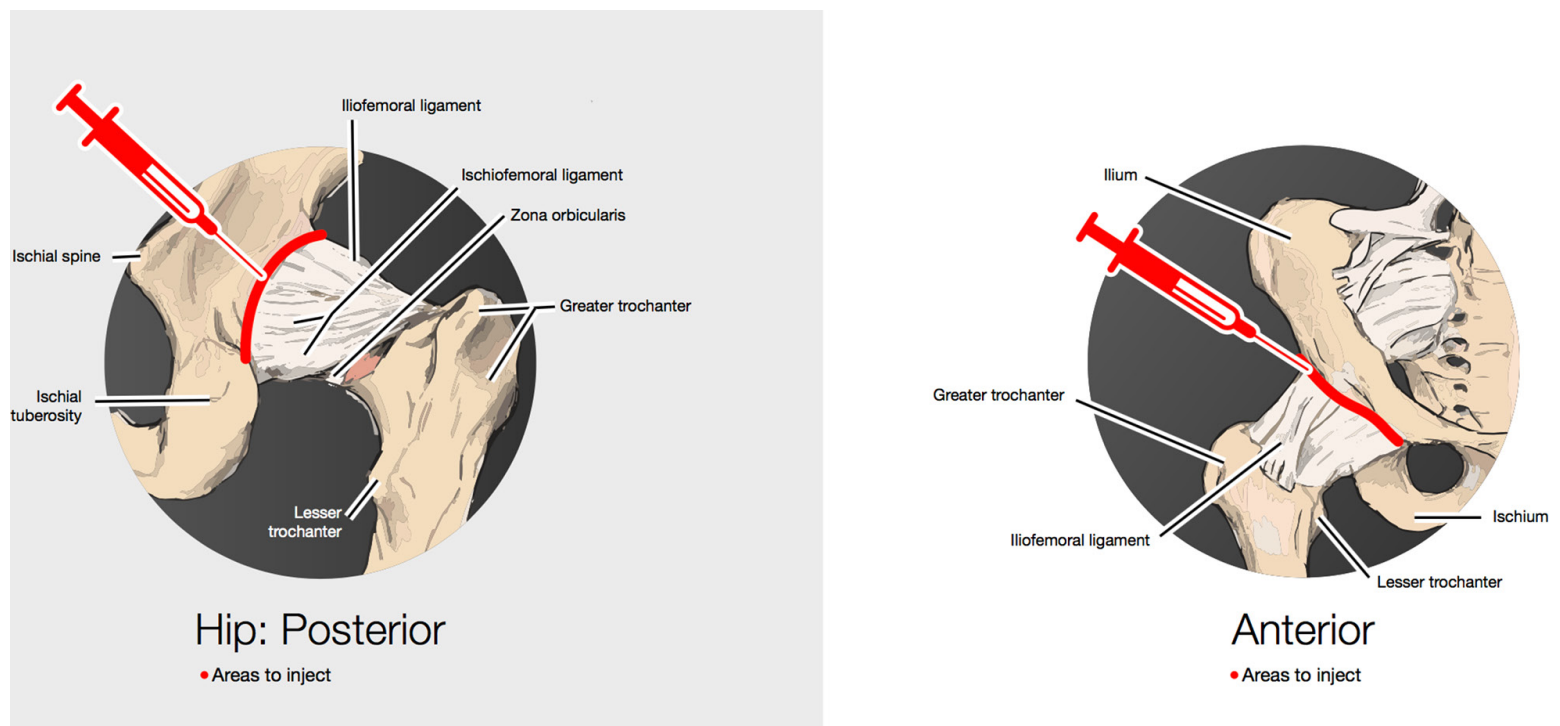

Figure 3 Examples for areas treated on a hip: injection is at the enthesis (in bone contact), on the side where the hip dislocates (usually posterior). Avoid going too low where the sciatic nerve runs. Inject where it is tender (in red). 


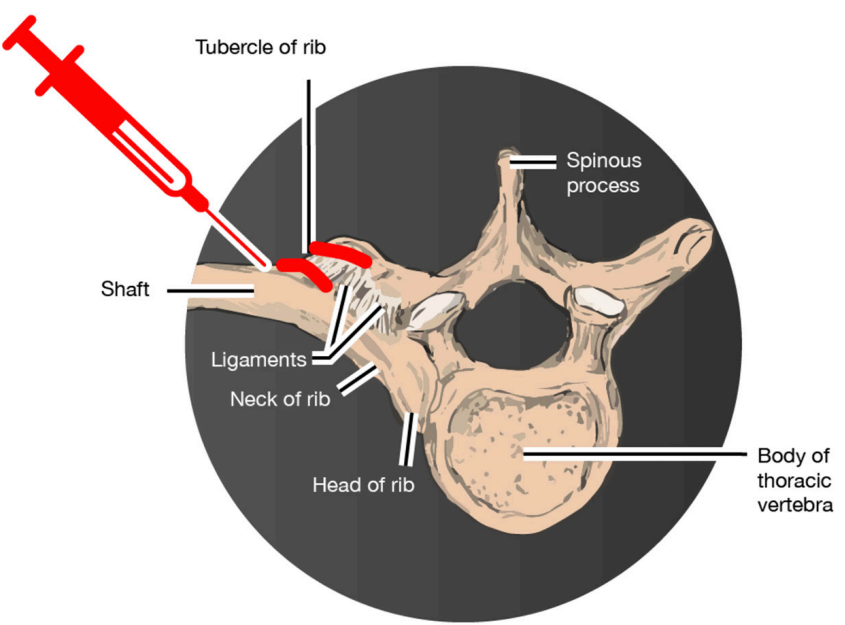

Rib Attachment

- Areas to inject

Figure 4 Example for area to treat on a rib: Start further out on the rib with a 2 -inch needle and slide in medially along the rib (to avoid missing the rib and hitting the lung).

value of 0.064 for null hypothesis (no difference). The cumulative probability graph (figure 5) shows the two groups are almost overlapping with minor differences likely from the variation in number size of each group.

\section{Side effects of treatments}

All injections were painful (if it is not tender, it is not in the right spot), with a bruised feeling that follows for $3-5$ days. Approximately 1:100 injections cause a significant bruise. There were no infections or other adverse events. Pain: While not being measured for this study, as these patients had other sources of pain that contribute to their total pain scores, the level of pain reduced and the patient ended up with little or no pain in the joints treated. Quality of life improved as well. Again, other injuries, and

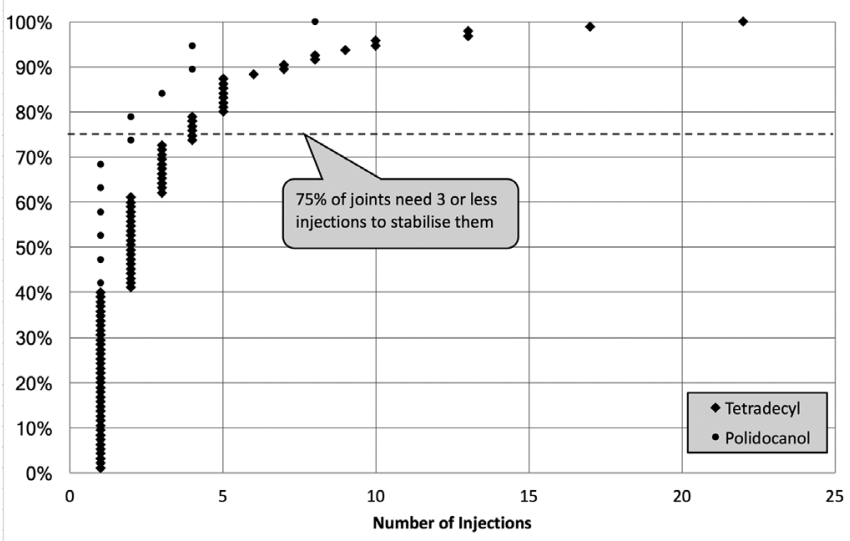

Figure 5 Cumulative probability graph for the number of injections needed to stabilise joints. $P=0.12$. The difference between the means is 1.2 (Cls -0.34 to 2.98 ). factors for EDS (mast cell activation syndrome, postural orthostatic tachycardic syndrome, gut dysmotility, urine frequency, and so on) contribute to the quality of life, or lack thereof, so measuring a difference that is specific to just the areas treated is difficult.

Note: Side effects from sclerosants at doses used in varicose veins and arteries (30-150 $\mathrm{mg} /$ dose) include: hypersensitivity, arterial and venous thrombosis, cardiac arrhythmias, visual/neurological disturbances and skin ulcerations. ${ }^{23}$ Differences in strength have known differences in effects. ${ }^{24-26}$ We have occasionally seen a rash, but we have not seen the rest of these side effects at this clinic with the low-dose (1.1-2.5 mg) treatments. We do avoid tetradecyl in those with a known sulfur allergy, and we avoid polidocanol in those with certain soap allergies.

\section{DISCUSSION}

Early analysis of treatments with strong strengthening injections of tetradecyl sulfate versus polidocanol indicates that they are likely equal to each other for efficacy. The study suggests that the process of building up collagen with sclerosants may make it harder to tear down a bundle than the effort required to tear down a few strands: even when dealing with abnormal collagen synthesis.

There is potential hope for the EDS community internationally with a non-surgical option for stabilising joints with results visible from quite low numbers of treatments needed per dislocated joint. If there is fresh injury, there is no significant contraindication to going back and treating the same area. There appear to be no additional risks for reinjecting areas that have been previously injected, when a new injury happens.

\section{Study limitations}

This is a retrospective, single-centre, single-physician study. Patient reporting is for no more dislocations (and unable to demonstrate dislocatability of joint(s) at will anymore). Follow-up was only from 3 months out to 3 years so far. There is the potential for reporting bias from patients with regard to further dislocation, or lack thereof, but the absence of being able to dislocate the joints at will at follow-up, and the willingness to have other joints treated when more than one was involved counters this risk to a certain degree.

However, there has been a high satisfaction rating from patients (and overloaded waiting list resulting from successful treatments of others). We have noted that subsequent reinjury takes fewer injections to restabilise the joints.

\section{Interpretation}

While the treatment of soft tissue injuries with prolotherapy/sclerosant therapy is not new, the use in the EDS population and more particularly in those with recurrent dislocations has not been previously studied. The use of sclerosants in TMJ injuries has been previously 
noted to be beneficial in the general population, and this study remains consistent with those results. ${ }^{22}$

\section{Generalisability}

More studies are needed internationally to confirm the results of this retrospective study. Other agents may also be considered, although our clinic found dextrose to be a much weaker sclerosant in general, and has not been used at our clinic for many years. If others can trial and reproduce these results, then there is a significant potential to reduce suffering in the EDS population. There remains potential that others with recurrent dislocations, but no EDS, may also benefit from this treatment.

Contributors FB planned the retrospective review, conducted the analysis and reported the work described in the article.

Funding The author has not declared a specific grant for this research from any funding agency in the public, commercial or not-for-profit sectors.

Competing interests FB reports personal fees from Rheumatology and Musculoskeletal Clinic during the conduct of the study.

Patient consent for publication Not required.

Ethics approval The Health and Disability Ethics Committees, Wellington, New Zealand, determined that formal ethics approval is not needed for this retrospective study.

Provenance and peer review Not commissioned; internally peer reviewed.

Data sharing statement These data are available from the author on reasonable request.

Open access This is an open access article distributed in accordance with the Creative Commons Attribution Non Commercial (CC BY-NC 4.0) license, which permits others to distribute, remix, adapt, build upon this work non-commercially, and license their derivative works on different terms, provided the original work is properly cited, appropriate credit is given, any changes made indicated, and the use is non-commercial. See: http://creativecommons.org/licenses/by-nc/4.0/.

\section{REFERENCES}

1. Am J Med Genet Part C Semin Med Genet C 2017:1-245.

2. Ericson WB, Wolman R. Orthopaedic management of the ehlersdanlos syndromes. Am J Med Genet C Semin Med Genet 2017:175:188-94.

3. Mitakides J, Tinkle BT. Oral and mandibular manifestations in the Ehlers-Danlos syndromes. Am J Med Genet C Semin Med Genet 2017:175:220-5.

4. Mann MW. Sclerotherapy: it is back and better. Clin Plast Surg 2011;38:475-87.

5. Cooley-Andrade O, Jothidas A, Goh WX, et al. Low-concentration detergent sclerosants stimulate white blood cells and release proinflammatory and proangiogenic cytokines in vitro. J Vasc Surg Venous Lymphat Disord 2014;2:433-40.

6. Burnes P. Sclerosant agents: indication, technique and endpoints. Springer New York: CardioVascular and Interventional Radiology, 2014: S88-S89.

7. Dirrichs T, Quack V, Gatz M, et al. Shear wave elastography (swe) for monitoring of treatment of tendinopathies: a double-blinded, longitudinal clinical study. Acad Radiol 2018;25:265-72.
8. Dagenais S, Haldeman S, Wooley JR. Intraligamentous injection of sclerosing solutions (prolotherapy) for spinal pain: a critical review of the literature. Spine $J$ 2005;5:310-28.

9. Hackett G. Ligament and Tendon Relaxation (Skeletal Disability) : Treated by Prolotherapy (Fibroosseous Proliferation).. 3rd edn. United States: Charles C Thomas Publisher, 1958: 1-151.

10. Cooper WM. Clinical evaluation of sotradecol, a sodium alkyl sulfate solution, in the injection therapy of varicose veins. Surg Gynecol Obstet 1946;83:647-52.

11. Soehendra N, Kempeners I. [Endoscopic control of gastrointestinal bleeding by sclerosing injections. Application to peptic ulcers and polypectomies (author's transl)]. Nouv Presse Med 1980;9:1955-6.

12. Sunding K, Willberg L, Werner S, et al. Sclerosing injections and ultrasound-guided arthroscopic shaving for patellar tendinopathy: good clinical results and decreased tendon thickness after surgery-a medium-term follow-up study. Knee Surg Sports Traumatol Arthrosc 2015;23:2259-68.

13. Hoksrud A, Bahr R. Ultrasound-guided sclerosing treatment in patients with patellar tendinopathy (jumper's knee). 44-month followup. Am J Sports Med 2011;39:2377-80.

14. Willberg L, Sunding K, Forssblad M, et al. Sclerosing polidocanol injections or arthroscopic shaving to treat patellar tendinopathy/ jumper's knee? A randomised controlled study. Br J Sports Med 2011:45:411-5

15. Ebrahimi SRS, Enamzadeh E, Babaei H. An evidence-based review of off-label uses of Polidocanol. Curr Clin Pharmacol 2017:12:223-30.

16. Branson R, Naidu K, du Toit C, et al. Comparison of corticosteroid, autologous blood or sclerosant injections for chronic tennis elbow. $J$ Sci Med Sport 2017;20:528-33.

17. Judson $\mathrm{CH}$, Wolf JM. Lateral epicondylitis: review of injection therapies. Orthop Clin North Am 2013;44:615-23.

18. Rabago D, Best TM, Zgierska AE, et al. A systematic review of four injection therapies for lateral epicondylosis: prolotherapy, polidocanol, whole blood and platelet-rich plasma. Br J Sports Med 2009;43:471-81.

19. Alfredson $\mathrm{H}$, Harstad $\mathrm{H}$, Haugen $\mathrm{S}$, et al. Sclerosing polidocanol injections to treat chronic painful shoulder impingement syndromeresults of a two-centre collaborative pilot study. Knee Surg Sports Traumatol Arthrosc 2006;14:1321-6.

20. Labra A, Haro-Valencia R, Huerta-Delgado A-D, et al. Efficacy of submucosal sodium tetradecyl sulfate in the soft palate as a treatment of the mild obstructive sleep apnea syndrome: a pilot study. Sleep Disord 2012;2012:1-4.

21. Brietzke SE, Mair EA. Injection snoreplasty: extended followup and new objective data. Otolaryngol Head Neck Surg 2003;128:605-15.

22. McCain JP, Hossameldin RH, Glickman AG. Preliminary clinical experience and outcome of the TMJ arthroscopic chemical contracture procedure in dislocation patients. J Oral Maxillofac Surg 2014;72:e16-17.

23. Khouri C, Roselli A, Roustit M. Safety profile of sclerosing agents: an analysis from the World Health organization pharmacovigilance database VigiBase ${ }^{\circledR}$. Fundamental and Clinical Pharmacology 2018;Jun 2018) suppl. Supplement 1:53.

24. Parsi K, Exner T, Low J, et al. In vitro effects of detergent sclerosants on clot formation and fibrinolysis. Eur J Vasc Endovasc Surg 2011;41:267-77.

25. Fisher DA. Regarding extensive tissue necrosis following high concentration sclerotherapy for varicose veins. Dermatol Surg 2000;26:1081.

26. Cooley-Andrade O, Cheung K, Chew A-N, et al. Detergent sclerosants at sub-lytic concentrations induce endothelial cell apoptosis through a caspase dependent pathway. Apoptosis 2016;21:836-45. 